Revista Arbitrada Interdisciplinaria de Ciencias de la Salud. SALUD Y VIDA

Volumen 5. Número 1. Año 5. Especial. 2021

Hecho el depósito de Ley: FA2016000010

ISSN: $2610-8038$

FUNDACIÓN KOINONIA (F.K)

Santa Ana de Coro, Venezuela.

Gabriela Liseth Vaca-Altamirano; Yuliana Guevara-Jaramillo; Stefano Espinoza-Guevara; Lady Mariela Trávez-Corrales

http://dx.doi.org/10.35381/s.v.v5i1.1623

\title{
Bases moleculares y genética en el cáncer oral
}

\section{Molecular basis and genetics in oral cancer}

\author{
Gabriela Liseth Vaca-Altamirano \\ ua.gabrielavaca@uniandes.edu.ec \\ Universidad Regional Autónoma de los Andes, Ambato \\ Ecuador \\ https://orcid.org/0000-0003-4707-7147 \\ Yuliana Guevara-Jaramillo \\ oa.yulianadgj79@uniandes.edu.ec \\ Universidad Regional Autónoma de los Andes, Ambato \\ Ecuador \\ https://orcid.org/0000-0002-0010-8412 \\ Stefano Espinoza-Guevara \\ oa.stefanooeg93@uniandes.edu.ec \\ Universidad Regional Autónoma de los Andes, Ambato \\ Ecuador \\ https://orcid.org/0000-0002-7592-9717 \\ Lady Mariela Trávez-Corrales \\ oa.ladymtc75@uniandes.edu.ec \\ Universidad Regional Autónoma de los Andes, Ambato \\ Ecuador \\ https://orcid.org/0000-0003-0196-4760
}

Recepción: 10 de agosto 2021

Revisado: 15 de septiembre 2021

Aprobación: 15 de noviembre 2021

Publicación: 01 de diciembre 2021 


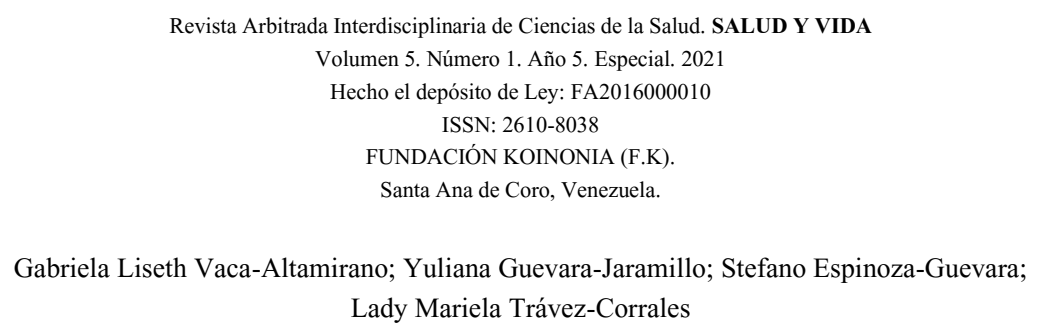

\title{
RESUMEN
}

Objetivo: Conocer las bases genéticas y moleculares que acompañan al cáncer oral. Método: De tipo documental, mediante una búsqueda de artículos en bases de datos como: Pubmed, Scielo, Dentistry and Oral, Science Direct. Resultados: Se han observado deleciones cromosómicas y mutaciones en los cromosomas 3p, 9p, 17p y 13q; se han descrito mutaciones en p53. Es posible que haya expresión excesiva de ciclina D1; a menudo también hay expresión exagerada del receptor para factor de crecimiento epidérmico. Conclusión: El mecanismo de muerte celular en especial la apoptosis, es un proceso fundamental y critico el cual determina la homeostasis del cuerpo, por lo general cuando existe un fallo a nivel de este proceso en cualquiera de sus etapas se va a producir una lesión.

Descriptores: Neoplasias de la Boca; radioterapia; diagnóstico por imagen. (Fuente: DeCS).

\begin{abstract}
Objective: To know the genetic and molecular basis of oral cancer. Method: Documentary type, through a search of articles in databases such as: Pubmed, Scielo, Dentistry and Oral, Science Direct. Results: Chromosomal deletions and mutations in chromosomes 3p, 9p, 17p and 13q have been observed; mutations in p53 have been described. Overexpression of cyclin D1 is possible; there is also often overexpression of the epidermal growth factor receptor. Conclusion: The mechanism of cell death, especially apoptosis, is a fundamental and critical process which determines the homeostasis of the body, generally when there is a failure at the level of this process in any of its stages an injury will occur.
\end{abstract}

Descriptors: Mouth Neoplasms; radiotherapy; diagnostic imaging. (Source: DeCS). 
Revista Arbitrada Interdisciplinaria de Ciencias de la Salud. SALUD Y VIDA

Volumen 5. Número 1. Año 5. Especial. 2021

Hecho el depósito de Ley: FA2016000010

ISSN: $2610-8038$

FUNDACIÓN KOINONIA (F.K)

Santa Ana de Coro, Venezuela.

Gabriela Liseth Vaca-Altamirano; Yuliana Guevara-Jaramillo; Stefano Espinoza-Guevara;

Lady Mariela Trávez-Corrales

\section{INTRODUCCIÓN}

Dentro de los tumores malignos el cáncer de cabeza y cuello presenta niveles desconcertantes teniendo una tasa de prevalencia en países sudamericanos asiáticos e indios llegando alcanzar hasta el $40 \%$ de todos los tumores malignos y esto sumado al hecho que el $3 \%$ tumores a nivel mundial se encuentran en boca hacen que el estudio de cáncer oral tome relevancia el estudio de las modificaciones genéticas (19). En base a lo mencionado anteriormente, la presente revisión bibliográfica tiene como propósito conocer las bases genéticas y moleculares que acompañan al cáncer oral.

\section{MÉTODO}

Se realizó una investigación de tipo documental, mediante una búsqueda de artículos en bases de datos como: Pubmed, Scielo, Dentistry and Oral, Science Direct. Fueron revisados un total de 50 artículos científicos que fueron obtenidos introduciendo los términos "cáncer oral" y "genética de la caries oral" en el periodo enero de 2000 a enero 2020; en los buscadores mencionados, una vez revisados los 60 artículos fueron seleccionados 28 ya que contenían la información necesaria para cumplir con el objetivo principal de esta revisión.

\section{RESULTADOS}

La cavidad bucal es una unidad funcional compuesta por varios órganos los cuales están asociados para realizar múltiples funciones como: la masticación, trituración, segregación de saliva la percepción del sabor, fonación y por supuesto el habla, cada uno de estos órganos son independientes pero con características propias diferenciables tanto morfológica como histológicamente, un aspecto destacable de cada uno de los órganos que compone la cavidad oral es su increíble adaptación relacionada a sus funciones específicas (1). 


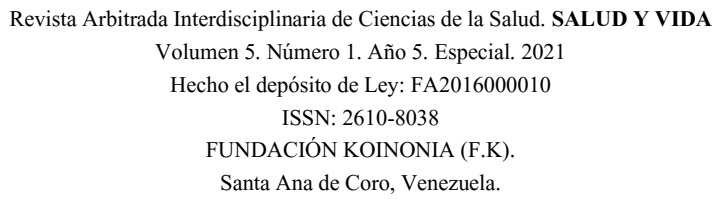

Gabriela Liseth Vaca-Altamirano; Yuliana Guevara-Jaramillo; Stefano Espinoza-Guevara;

Lady Mariela Trávez-Corrales

Por otro lado, el VIH tiene una estrecha relación con el cáncer oral especialmente en los pacientes que desarrollan linfoma el cual básicamente se desarrolla a nivel de ganglios linfáticos y este tipo de linfoma tiene repercusión en el sistema inmunológico del paciente, así como en otros factores. Los pacientes con una etapa avanzada de VIH y en pacientes que no presentan tratamiento antirretroviral, tienen un bajo recuento de CD4, estos CD4 o linfocitos TCD4 son células defensivas importante para los procesos apoptóticos y de ciclo celular normal una falla en estas células puede dar origen a la génesis del cáncer y en la proliferación de este (2)(3)(4).

El virus del papiloma humano (VPH) presenta una relación estrecha con el cáncer oral en específicamente los subtipos de VPH 16 y 18 tiene una tendencia a la generación de oncogenes los cuales van a estar directamente relacionados con falles a nivel del ciclo celular las cuales van a repercutir en la aparición y proliferación de diferentes tipos de tumores malignos a nivel oral teniendo una prevalencia significativa en el cáncer orofaringe (5).

El microambiente de la mucosa oral proporciona la adaptabilidad a estas células al dotar de ventajas de proliferación para la supervivencia en relación a células normales en su epitelio, la génesis de la tumoración está condicionada por varios factores como el anticrecimiento, la autosuficiencia en las señales de crecimiento, las señales de crecimiento, falta de sensibilidad a las señales, un potencial replicativo ilimitado, la capacidad de evadir la apoptosis aumento de la angiogénesis invasión y metástasis (6).

La cancerización de campo es un término que hace referencia al desarrollo del cáncer en distintas zonas del tejido de la mucosa oral (7) que se ha visto evidenciado en tejido conformado tanto por epitelio escamoso y de transición, el carcinoma en otros tejidos se desarrollan en periodos prolongados durante los cuales se producen múltiples sitos de transmutación neoplásica, a partir de varios estudios se ha demostrado la alteración del p53 en leucoplasia premaligna y carcinoma en la misma cavidad oral (6), creando una reducción de la actividad supresora tumoral característica de este gen, la continua 
mutación representa cambios en la reparación del ADN y la apoptosis lo que va a derivar un aumento la susceptibilidad para futuras transformaciones, la recopilación de datos afirma que el cáncer que prolifera en sitios distantes a nivel oral son derivados de una alteración celular inicial (9).

El ciclo celular es un proceso complejo y preciso en el cual se regula la división celular y es fundamental para la generación de células nuevas que remplazan a células dañadas en un organismo adulto (10) la ejecución de los eventos del ciclo celular toman lugar en cuatro fases: teniendo en cuenta la fase Go que se define como una fase de reposos de la célula en la cual no se divide pero tiene la capacidad de reiniciar el ciclo celular (11) en la fase G1 que es una fase de crecimiento se inicia con una celular ya dividida previamente en esta fase la célula tiene la capacidad de crecer y producir absolutamente todas las proteínas necesarias para la síntesis del ADN se continua en la fase $\mathbf{S}$ en la cual se suplica en ADN y al finalizar la fase el núcleo tendrá el doble de ADN y proteínas nucleares en la fase $\mathbf{G}_{2}$ sintetiza $A R N$ y proteínas, al incrementar las proteínas y organelos la célula aumenta de tamaño y el núcleo se organiza para la división celular a estas tres fases $\left(G_{1}, S\right.$ y $\left.G_{2}\right)$ se denomina interfase posterior a ello la célula inicia la fase de mitosis en esta apartado del ciclo celular se da la separación de cromosomas, división nuclear y citocinesis (12).

A nivel de muerte celular existen dos procesos uno de ellos necrosis el cual es el resultado último de la lesión celular, es uno de los acontecimientos más cruciales en la evolución de la enfermedad de cualquier tejido u órgano. Es el resultado de diversas causas, incluyendo isquemia (falta de riego sanguíneo), infección, toxinas y reacciones inmunitarias. La muerte celular es, además, una función normal y esencial en la embriogénesis, como una parte del desarrollo de órganos y el mantenimiento de la homeostasia (13).

La angiogénesis la cual se define como el fenómeno de producción de nuevos vasos sanguíneos a partir de los preexistentes, es un determinante importante en la aparición 
del carcinoma ya que proporciona al tumor nutrición y permite su proliferación (14). El factor de crecimiento vascular endotelial (VEGF) es el mecanismo que regula este proceso, y el gen que lo codifica actúa como un oncogén en el cáncer oral permitiendo así el crecimiento vascular, la nutrición del tejido carcinómico, así como la proliferación del tumor. El tejido tumoral presenta estímulos hipóxicos (insuficiencia de oxígeno) los cuales dispara la producción del factor de crecimiento vascular endotelial que aumentara la microdensidad vascular (15)(16).

A nivel del tejido se puede evidenciar que las células tumorales superan la resistencia que ofrece el tejido especifico en el que se esté desarrollando el carcinoma, y esta característica es un mecanismo desplegado por las células neoplásicas, misma función que esta mediada por las acciones de las metaproteínas de matriz (MMP), un grupo de enzimas con gran cantidad de zinc las cuales son capaces de degradar la matriz extracelular (16).

Muchas de estas enzimas se presentan en el cáncer oral y juegan un papel fundamental para la proliferación y expansión del tumor en el epitelio, es así que la expresión a nivel inmunológico, histológico, y bioquímico tanto del MMP-2 como del MMP-9 se ha relacionado con el potencial invasivo, y como resultados se arroja el hecho de que el MMP-2 está asociado a capacidades metastásicas de ganglios linfáticos, y cabe destacar que un fator exógenos como es el alcohol participa en la carcinogénesis oral a través de estímulos a nivel de las metaproteínas de matriz MMP (17).

La activación de los genes supresores se va a encargar de la protección de células cuando adquieren propiedades malignas, actúan a nivel del chequeo del ciclo celular (mecanismo molecular encargado de cumplir condiciones que permitan el paso de un clico celular al otro). La proteína retinoblastoma (pRb) es una proteína supresora de tumores que se encuentra modificada en diferentes tipos de cáncer como el de pulmón, próstata, mama y entre ellos el cáncer oral (18). 
La proteína retinoblastoma en el estado hipofosforilado, es decir cuando es apta para realizar su función junto con miembros de su familia como p107 y p130 accede a la activación del factor de transcripción E2F teniendo un papel importante en la progresión del ciclo celular en la fase $\mathrm{G} 1$ a $\mathrm{S}$, los porcentajes altos que tienen tanto las lesiones premalignas $(64 \%)$ y carcinomas orales $(70 \%)$ se manifiesta por la omisión de pRb, interpretando como la ausencia de las funciones que regulan el ciclo celular que están unidas a la proteína retinoblastoma, interpretándose como la falta de la función que regula el ciclo ligadas a esta proteína, un alto índice de alteraciones en los miembros de pRb se presentan en los carcinomas (19).

Hay genes que tiene una acción supresora tumoral específicamente los genes que codifican a las quinasas dependientes de las ciclinas, estas promueven un importante funcionamiento de la proteína retinoblastoma y por lo tanto un adecuado control del ciclo celular, el gen CDKN2A (inhibidor de la quinasa dependiente de ciclina 2A) el cual codifica importantes proteínas como ejemplo a la p16 (proteína supresora de tumores (20), la cual se va a ubicar específicamente en el locus 9p21 la cual es una zona muy vulnerable del genoma humano en estudios y cáncer bucal. En el $80 \%$ de esta afección se evidencia una falta de función de p16, por lo que se puede inferir una importancia de esta proteína para el mantenimiento de un equilibrio celular, y la importancia del gen CDKN2A (21). Otro gen supresor tumoral TP53, la supresión de las funciones de este gen así como su sistema molecular se observa en numerosas lesiones cancerosas la proteína p53 tiene la capacidad de detener completamente el ciclo celular si halla alguna alteración a nivel del ADN para posteriormente establecer mecanismos para su reparación y si el daño es irreversible el p53 induce a la apoptosis celular, el sistema p53 tiene la función de eliminar la posibilidad de la replicación del ADN lesionado en la que nuevos eventos oncogénicos puede llevar a la aparición y proliferación de una lesión maligna (22).

En estas regiones se presentan genes reguladores del ciclo celular los cuales son importantes para el mantenimiento de la homeostasis, ante una alteración en estas 
regiones las células adquieren ventajas proliferativas, estas ventajas a nivel de las células epiteliales de la mucosa oral crean un campo invasivo precanceroso que se expande progresivamente remplazando al epitelio normal típico, todas las células presentan características genéticas alteradas, cabe destacar que estos campos son expansivos mas no invasivos, adquieren capacidades invasivas cuando existen oncogenes avanzados producidos por las alteraciones cromosómicas en regiones específicas como $8 p, 13 q$ y 18q. Adquiere relevancia la diferenciación entre segundos tumores primarios los cuales tiene un perfil genético diferente al tumor inicial y los tumores de campo que ha diferencia comparte con el tumor inicial (23).

El diagnóstico del cáncer oral en estadios tempranos o precáncer conlleva un reto para los científicos en la actualidad, sin embargo, mediante varias investigaciones se ha evidenciado que algunos biomarcadores salivales se han visto alterados es el caso de Cytokeratin tanto como el CK13, CK19 y SCC (Biomarcador de carcinoma de células escamosas). Estudios han demostrado una alta expresión de mRNA de estas proteínas lo que a su vez indica aparición de cáncer, dentro de este grupo de marcadores el CK19 se ha demostrado que son los más fiables para la detención de cáncer a pesar de que el SCCA es un marcador fiable para diagnóstico de metástasis (24)(25).

El estadio primario de una lesión cancerosa clínicamente es asintomático, existen manifestaciones relevantes en estadios avanzados, es así que destacamos síntomas como, ulceraciones, perdida de la función de varias estructuras, movilidad de las piezas y perdida de las mismas, dificultad y dolor en la deglución, tumoraciones en el cuello (26) aparición de leucoplasia que se manifiesta con aspecto de color blanco, eritroplasia manifiesta con aspecto de color blanco también se puede visualizar una mancha de color blanca y color roja denominada eritroleucoplasia, estas últimas manifestaciones con el tiempo se ulceran y con el tiempo evolucionan a una masa exofítica - endofítica entre otras manifestaciones se pueden presentar una sensación de cosquilleo en lengua y 
labios, crecimiento papilar, si las alteraciones anteriormente expuestas se mantiene por más de 3 semanas en la cavidad se puede sospechar de cáncer.

Las lesiones de cáncer a pesar de pueden afectar a cualquier parte de la cavidad oral tiene una prevalencia en ciertas zonas como a nivel del suelo de la boca en el borde lateral de la lengua, orofaringe, paladar blando, mucosa de los carrillos y labio inferior, en pacientes que son fumadores frecuentes e ingieren alcohol las lesiones se presentan mayormente en mucosa oral y alveolos (27).

Para el diagnóstico de una lesión cancerosa es esencial buscar y determinar los factores de riesgo que propician el desarrollo de la lesión a nivel oral en paciente fumadores el riesgo aumenta de cinco a nueve veces que un no fumador por otro lado si en personas que consumen alcohol frecuente el riesgo aumenta treinta veces más para el cáncer oral o faríngeo y para pacientes que presentan ambos hábitos el riesgo incrementa cien veces de lo normal, una exploración clínica oportuna y eficaz toma relevancia al momento de identificar si una lesión cancerosa. Uno de los métodos más efectivos para la detención de una lesión cancerosa en estados tempranos o tardíos es un conjunto de técnicas que desde nuestra perspectiva son relevantes refiriéndonos a la biopsia cuando una lesión nos hace sospechar que puede producir magnificación (28).

\section{CONCLUSIONES}

Destacamos de nuestra investigación fundamentalmente a los genes supresores tumorales, así como los genes implicados en las alteraciones a nivel del ciclo celular que produce cáncer oral, en relación a los genes supresores tumorales podemos inferir que están implicados en los procesos de división celular específicamente en la programación de la muerte celular, regulación de expresión genética, estabilidad del genoma, control del ciclo celular, la perdida de la función de estos genes provoca un descontrol a nivel del ciclo celular y de la apoptosis generando así la aparición de cáncer con la proliferación descontrolada del crecimiento celular. 


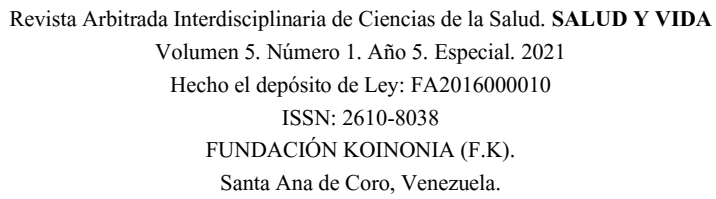

Gabriela Liseth Vaca-Altamirano; Yuliana Guevara-Jaramillo; Stefano Espinoza-Guevara;

Lady Mariela Trávez-Corrales

El gen retinoblastoma es el principal gen supresor tumoral el cual está estrechamente relacionado con la aparición de cáncer actuando en el ciclo celular específicamente en la fase $\mathrm{G} 1$ - S este gen en lesiones de cáncer bucal se ve alterado provocando así que las células que deberían ser suprimidas pasen a la siguiente fase del ciclo celular (fase S). Otra alteración relevante son las aberraciones genéticas que se da a nivel cromosómico en donde se ha descrito que alteraciones a nivel de los genes en los cromosomas $3 p, 9 p$ y $17 p$ ya sea por heterocigosidad o delección condicionan a la aparición de tumores.

Un determinante de la aparición de las lesiones tumorales se debe al desarrollo de los oncogenes que básicamente son genes que a partir de su actividad generan un mecanismo molecular el cual va a incidir directamente en la transformación maligna de la célula, así como la proliferación de células aberrantes un ejemplo es el receptor que codifica el factor de crecimiento (ErbB).

Se han observado deleciones cromosómicas y mutaciones en los cromosomas 3p, 9p, 17p y 13q; se han descrito mutaciones en p53. Es posible que haya expresión excesiva de ciclina D1; a menudo también hay expresión exagerada del receptor para factor de crecimiento epidérmico.

Se describió el mecanismo de muerte celular en especial la apoptosis la cual es un proceso fundamental y critico el cual determina la homeostasis del cuerpo, por lo general cuando existe un fallo a nivel de este proceso en cualquiera de sus etapas se va a producir una lesión.

Destacamos los principales genes que están implicados en el desarrollo del carcinoma bucal identificando sus posibles alteraciones que conllevan a un desequilibrio en los procesos de muerte celular y de identificación de células defectuosas, para así comprender mejor la aparición a partir de los componentes genéticos y moleculares, cae destacar que a estos factores genéticos y moleculares se le adiciona factores exógenos como alcohol y tabaco determinantes para la aparición y progresión de la lesión. 
Revista Arbitrada Interdisciplinaria de Ciencias de la Salud. SALUD Y VIDA

Volumen 5. Número 1. Año 5. Especial. 2021

Hecho el depósito de Ley: FA2016000010

ISSN: $2610-8038$

FUNDACIÓN KOINONIA (F.K)

Santa Ana de Coro, Venezuela.

Gabriela Liseth Vaca-Altamirano; Yuliana Guevara-Jaramillo; Stefano Espinoza-Guevara;

Lady Mariela Trávez-Corrales

\section{CONFLICTO DE INTERÉS}

Los autores declaran que no tienen conflicto de interés en la publicación del artículo.

\section{FINANCIAMIENTO}

No monetario.

\section{AGRADECIMIENTO}

A la Universidad Regional Autónoma de los Andes; por apoyar el desarrollo de la investigación.

\section{REFERENCIAS}

1. García-García V., Bascones Martínez A. Update in oral cancer. Av Odontoestomatol [Internet]. 2009; 25(5): 239-248.

2. Betancourt-Gambino J. Cáncer en pacientes viviendo con $\mathrm{VIH} /$ sida [Cancer in patients living with HIV/AIDS]. Rev Cubana Med Gen Integr [Internet]. 2018; 34(2): $1-9$.

3. Cameron JE, Hagensee M. HPV-Associated Oropharyngeal Cancer in the HIV/AIDS Patient. Cancer Treat Res. 2019;177:131-181. doi:10.1007/978-3-030$\underline{03502-0 \quad 6}$

4. McNally GA. HIV and Cancer: An Overview of AIDS-Defining and Non-AIDSDefining Cancers in Patients With HIV. Clin J Oncol Nurs. 2019;23(3):327-331. doi:10.1188/19.CJON.327-331

5. Spano JP, Veyri M, Gobert A, et al. Immunotherapy for cancer in people living with HIV: safety with an efficacy signal from the series in real life experience. AIDS. 2019;33(11):F13-F19. doi:10.1097/QAD.0000000000002298

6. Merlo LM, Pepper JW, Reid BJ, Maley CC. Cancer as an evolutionary and ecological process. Nat Rev Cancer. 2006;6(12):924-935. doi:10.1038/nrc2013 
Revista Arbitrada Interdisciplinaria de Ciencias de la Salud. SALUD Y VIDA

Volumen 5. Número 1. Año 5. Especial. 2021

Hecho el depósito de Ley: FA2016000010

ISSN: $2610-8038$

FUNDACIÓN KOINONIA (F.K)

Santa Ana de Coro, Venezuela.

Gabriela Liseth Vaca-Altamirano; Yuliana Guevara-Jaramillo; Stefano Espinoza-Guevara;

Lady Mariela Trávez-Corrales

7. García-García V, González-Moles MA, Bascones Martínez A. Bases moleculares del cáncer oral: Revisión bibliográfica [Molecular basis of oral cancer: literature review.]. Av Odontoestomatol [Internet]. 2005 ; 21(6): 287-295.

8. Gallegos-Villanueva MJ, Chimenos-Küstner E, López-López J, Roselló-Llabrés X. Cancerización de campo: revisión del concepto [Field cancerization: revision of the concept]. Av Odontoestomatol [Internet]. 2007; 23(1): 35-44.

9. Waldum HL, Fossmark R. Types of Gastric Carcinomas. Int J Mol Sci. 2018;19(12):4109. Published 2018 Dec 18. doi:10.3390/ijms19124109

10. Dai L, Zhao T, Bisteau X, et al. Modulation of Protein-Interaction States through the Cell Cycle. Cell. 2018;173(6):1481-1494.e13. doi:10.1016/j.cell.2018.03.065

11. Kartal-Yandim M, Adan-Gokbulut A, Baran Y. Molecular mechanisms of drug resistance and its reversal in cancer. Crit Rev Biotechnol. 2016;36(4):716-726. doi:10.3109/07388551.2015.1015957

12. Karimian A, Ahmadi Y, Yousefi B. Multiple functions of p21 in cell cycle, apoptosis and transcriptional regulation after DNA damage. DNA Repair (Amst). 2016;42:6371. doi:10.1016/i.dnarep.2016.04.008

13. Fricker M, Tolkovsky AM, Borutaite V, Coleman M, Brown GC. Neuronal Cell Death. Physiol Rev. 2018;98(2):813-880. doi:10.1152/physrev.00011.2017

14. Viallard C, Larrivée B. Tumor angiogenesis and vascular normalization: alternative therapeutic targets. Angiogenesis. 2017;20(4):409-426. doi:10.1007/s10456-017$\underline{9562-9}$

15. Czarnecka AM, Niedzwiedzka M, Porta C, Szczylik C. Hormone signaling pathways as treatment targets in renal cell cancer (Review). Int $\mathrm{J}$ Oncol. 2016;48(6):2221-2235. doi:10.3892/ijo.2016.3460

16. Ramjiawan RR, Griffioen AW, Duda DG. Anti-angiogenesis for cancer revisited: Is there a role for combinations with immunotherapy?. Angiogenesis. 2017;20(2):185-204. doi:10.1007/s10456-017-9552-y 


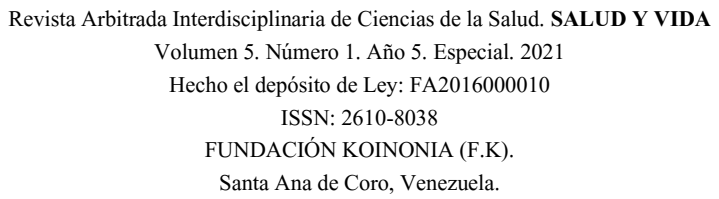

Gabriela Liseth Vaca-Altamirano; Yuliana Guevara-Jaramillo; Stefano Espinoza-Guevara;

Lady Mariela Trávez-Corrales

17. Sajib S, Zahra FT, Lionakis MS, German NA, Mikelis CM. Mechanisms of angiogenesis in microbe-regulated inflammatory and neoplastic conditions. Angiogenesis. 2018;21(1):1-14. doi:10.1007/s10456-017-9583-4

18. Wong T, Wiesenfeld D. Oral Cancer. Aust Dent J. 2018;63 Suppl 1:S91-S99. doi:10.1111/adj.12594

19. Ter Huurne M, Peng T, Yi G, van Mierlo G, Marks H, Stunnenberg HG. Critical Role for P53 in Regulating the Cell Cycle of Ground State Embryonic Stem Cells. Stem Cell Reports. 2020;14(2):175-183. doi:10.1016/j.stemcr.2020.01.001

20. Baker SJ, Vogelstein B. p53: a tumor suppressor hiding in plain sight. $J$ Mol Cell Biol. 2019;11(7):536-538. doi:10.1093/jmcb/miz068

21. Weinhold N, Ashby C, Rasche L, et al. Clonal selection and double-hit events involving tumor suppressor genes underlie relapse in myeloma. Blood. 2016;128(13):1735-1744. doi:10.1182/blood-2016-06-723007

22. Feng LY, Chen CX, Li L. Hypermethylation of tumor suppressor genes is a risk factor for poor prognosis in ovarian cancer: A meta-analysis. Medicine (Baltimore). 2019;98(8):e14588. doi:10.1097/MD.0000000000014588

23. Schoenberg M, Cairns $P$, Brooks JD, et al. Frequent loss of chromosome arms $8 p$ and $13 q$ in collecting duct carcinoma (CDC) of the kidney. Genes Chromosomes Cancer. 1995;12(1):76-80. doi:10.1002/gcc.2870120115

24. Khurshid Z, Zafar MS, Khan RS, Najeeb S, Slowey PD, Rehman IU. Role of Salivary Biomarkers in Oral Cancer Detection. Adv Clin Chem. 2018;86:23-70. doi:10.1016/bs.acc.2018.05.002

25. Varela-Centelles P, Castelo-Baz P, Seoane-Romero J. Oral cancer: Early/delayed diagnosis. Br Dent J. 2017;222(9):643. doi:10.1038/sj.bdj.2017.380

26. Chattopadhyay I, Verma M, Panda M. Role of Oral Microbiome Signatures in Diagnosis and Prognosis of Oral Cancer. Technol Cancer Res Treat. 2019;18:1533033819867354. doi: $10.1177 / 1533033819867354$ 
27. Mateo-Sidrón Antón M.C., Somacarrera Pérez M.L. Cáncer oral: genética, prevención, diagnóstico y tratamiento. revisión de la literatura [Oral cancer: Genetics, prevention, diagnosis and treatment. Literature review]. Av Odontoestomatol [Internet]. 2015; 31(4): 247-259. Disponible en: http://scielo.isciii.es/scielo.php?script=sci_arttext\&pid=S0213$12852015000400002 \& \operatorname{lng}=$ es. 12852015000400002.

28. Figueroa-Sánchez M, Núñez-Atahualpa L, Hernández-Zúñiga M, et al. Evaluación clínica y ultrasonográfica de la glándula tiroides en pacientes con artritis reumatoide [Clinical and ultrasonographic evaluation of the thyroid gland in patients with rheumatoid arthritis]. Gac Med Mex. 2018;154(4):432-437. doi:10.24875/GMM.18003556

2021 por los autores. Este artículo es de acceso abierto y distribuido según los términos y condiciones de la licencia Creative Commons Atribución-NoComercial-Compartirlgual 4.0 Internacional (CC BY-NC-SA

4.0) (https://creativecommons.org/licenses/by-nc-sa/4.0/). 